$4 \quad$ P.M. Berry ${ }^{1}$, C.J. Baker ${ }^{2}$, D. Hatley ${ }^{1}$, R. Dong ${ }^{3}$, X. Wang ${ }^{3}$, G.A. Blackburn ${ }^{4}$, Y. Miao ${ }^{5}$, M.

$5 \quad$ Sterling $^{2}$, J.D. Whyatt ${ }^{4}$

\section{Development and application of a model for calculating the risk of stem and root}

\section{lodging in maize}

6

1 ADAS High Mowthorpe, Malton, North Yorkshire, YO178BP, UK

2 School of Engineering, the University of Birmingham, Edgbaston, Birmingham B15

$2 T T, U K$

3 College of Resources and Environment Sciences, China Agricultural University, Beijing 100193, China

4 Lancaster Environment Centre, Lancaster University, Lancaster, LA1 4YQ, UK

5 Department of Soil, Water and Climate, University of Minnesota, St. Paul, MN 55108

\title{
Abstract
}

Lodging is a major constraint to increasing the global productivity of maize (Zea Maize L.). The objectives of this paper are to: i) describe a model for stem and root lodging in maize, ii) calibrate the anchorage strength component of the model, iii) evaluate the model's applicability by assessing its capacity to explain effects of crop husbandry on lodging risk and iv) investigate the potential to further develop the lodging model to predict lodging risk at an early enough growth stage for tactical agronomic action to minimise lodging risk. The study involved a 
multidisciplinary collaboration between crop scientists, wind engineers and geospatial scientists in the UK and China. Three field experiments with plant population density and nitrogen $(\mathrm{N})$ fertiliser rate treatments were conducted in the UK and China to develop and test the lodging model. Plant characteristics associated with lodging were measured in the experiments after flowering. An existing model of cereal anchorage strength that uses the spread of the root plate as its primary input was demonstrated to be applicable for maize and calibrated for this crop species. The lodging model's predictions of the effects of plant population and $\mathrm{N}$ fertiliser on lodging risk were consistent with published observations. The lodging model calculated that increasing the plant population significantly reduced the anchorage and stem failure wind speeds in all experiments, thus increasing the risk of lodging. This effect was primarily due to increased plant population reducing the spread of the root plate and the stem strength. Changes in $\mathrm{N}$ fertiliser had a smaller effect on the lodging associated plant characters. A sensitivity analysis showed that stem failure wind speed was influenced most by variation in stem strength and root failure wind speed was influenced most by variation in the spread of the root plate. This study has shown that the leaf area index measured at leaf 4, 6 or 8 stages is a good indicator of a crop's future risk of lodging, which demonstrates the potential to develop the model into a practical tool for predicting lodging risk in time for tactical agronomic decisions to be made during the crop's growing period. 
Lodging is defined as the permanent displacement of plant stems from their vertical position

(Berry et al., 2004). Lodging is a major problem in Maize (Zea Mays L.) and has been estimated to account for global yield losses of between 5\% and 20\% per year for this crop (Flint-Garcia et al., 2003; Hu et al., 2013). This amounts to a cost of lost production of approximately $\$ 7.5$ to $\$ 30$ billion per year based on gross production figures in 2004-6 (http://www.fao.org/home/en/). It has been reported that lodging can reduce maize yield by 14$28 \%$ when it occurs during the 12 -leaf stage, and by $30-48 \%$ when it occurs during grainfilling ( $\mathrm{Li}$ et al., 2015a, b). In addition to yield loss, lodging reduces grain quality and increases the time to harvest and drying costs (Kamara et al. 2003; Huang et al. 2015). Lodging is a particular challenge in maize because increasing plant density increases both yield and lodging susceptibility (Xue et al., 2017).

Maize lodging has been shown to result from buckling of the stem (stem lodging) (Hu et al., 2013) or failure of the anchorage system (root lodging) (Fincher et al. 1985; Kamara et al. 2003). Previous studies of maize lodging have generally focussed on specific components of the lodging process, e.g. the stem strength or rind penetration resistance (Colbert et al., 1984; Li et al., 2014), or have not accounted for the dynamic nature by which the plant interacts with the wind (Guo et al., 2019) or rely on generating artificial wind with a mobile wind machine which does not take into account the appropriate turbulence characteristics of the wind (Wen et al., 2019). However, to fully understand how factors influence both stem and root lodging it is necessary to integrate all the key processes including: the dynamic interactions between the plant and wind, the strength of the stem and the strength of the anchorage system. A comprehensive lodging model has been successfully developed for cereal plants (Baker et al., 2014; Berry et al., 2003). This model assumes that the unit of lodging is a single plant and stem lodging is expected if the wind-induced bending moment 
(leverage) of the shoot exceeds the stem failure moment, and root lodging is expected if the leverage force of the plant exceeds the anchorage failure moment. Recently the aerodynamic properties of plants have been ascertained experimentally (Joseph et al., 2020) which enables the cereal lodging model to be developed to better account for interactions between the wind and the plant for a range of plant species including maize. However, to date there is no satisfactory description of the lodging process in maize that accounts for all the key processes: plant/wind interaction, stem strength and anchorage strength.

The aim of this paper is to develop a realistic model of lodging in maize that can be used to quantify the effects on lodging risk of agronomic lodging control approaches without relying on the occurrence of natural lodging. This understanding will help farmers and crop advisors develop agronomic strategies for minimising the lodging risk of future crops, for example by optimising cultivar choice and seed rate. A second application of a lodging model is to help farmers make in-season, or tactical, agronomic decisions to minimise lodging risk based on observations of the growing crop. Examples of tactical decisions are changing the rate and timing of nitrogen $(\mathrm{N})$ fertiliser or the use of plant growth regulators to shorten the crop. The lodging associated crop characteristics used as the lodging model inputs have not yet developed when tactical decisions about remedial treatments must be made. Therefore, it will be necessary to identify surrogate crop parameters that are reliable indicators of the values of lodging associated plant characteristics. A previous study of oilseed rape (Brassica napus L.) has demonstrated that the green area index of a crop at the start of stem extension is a useful indicator of its future lodging risk (Berry and Spink, 2009). It is therefore possible that early season canopy size may also be a useful indicator of future values of the lodging associated plant characters in other crops such as maize.

The objectives of this paper are to: i) describe a model for stem and root lodging in maize, ii) calibrate the anchorage strength component of the model, iii) evaluate the model's 
applicability by assessing its ability to explain effects of crop husbandry on lodging risk and iv) investigate the potential to further develop the lodging model to predict lodging risk at an early enough growth stage for tactical agronomic action to minimise lodging risk.

\section{Model of maize lodging}

Aerodynamic investigations of the maize shoot have shown that it behaves as a damped harmonic oscillator after being subjected to wind loading (Joseph et al., 2020). Therefore, the mechanistic process of lodging in maize can be modelled in the same way as other plant species with similar aerodynamic points of structural failure (e.g. wheat). A generalised model used to describe the lodging process in cereals (Baker et al., 2014; Berry et al., 2003) can therefore be adapted for maize, taking account of maize-specific calibrations estimated by Joseph et al. (2020). The maize lodging model assumes that the unit of lodging is a single plant and stem lodging is expected if the wind-induced bending moment (leverage of the shoot) exceeds the stem failure moment (stem strength at the point of failure), while root lodging is expected if the leverage force exceeds the anchorage failure moment (anchorage strength at the point of failure). It is assumed that stem failure results from buckling of the stem, for which the mechanical properties of a cylinder apply. The mechanism of anchorage failure in maize has been shown to be similar to wheat (Ennos et al. (1993). Therefore, failure moment of the anchorage system is assumed to be proportional to the product of the spread of the crown roots cubed and the shear strength of the surrounding soil, similar to anchorage models for wheat (Baker et al., 1998), sunflower (Sporoso et al. 2008; 2010) and oats (Mohammadi et al., 2020)

The bending moment $(B)$ at any point along the shoot is obtained as a function of mean wind speed $\bar{U}$, using the density of air $\left(\rho=1.2 \mathrm{~kg} / \mathrm{m}^{3}\right)$, the drag area of the $\operatorname{shoot}\left(A_{C F}=0.153 \mathrm{~m}^{2}\right)$, 
the shoot's height at centre of gravity $(X)$, the shoot's natural frequency $\left(f_{n}\right)$, the acceleration due to gravity $\left(g=9.81 \mathrm{~m} \mathrm{~s}^{2}\right)$, the turbulence intensity $\left(I_{u}\right)$ and the shoot's damping ratio $(\theta=$ 0.13). Values for $A_{C F}, X, I_{u}$ and $f_{n}$ were experimentally determined for maize by Joseph et al. (2020).

$B=\frac{0.5 \rho A C_{F} \bar{U}^{2}\left(1+\left(2 \pi f_{n}\right)^{2}(X / g)\right)}{\left(2 \pi f_{n}\right)^{2}(X / g)}\left(\cos \left(\frac{\alpha l}{h}\right)-\cot \alpha \sin \left(\frac{\alpha l}{h}\right)\right)\left(1+6.86 I_{u}\left(1+0.366\left(\frac{\pi}{4 \theta}\right)\right)^{0.5}\right)$

In the above equation, $l$ and $h$ represent the height above the ground at which the bending moment is considered and the total height of the shoot respectively; $\alpha$ is a constant determined from the relationship:

$\left(2 \pi f_{n}\right)^{2}(X / g)=\frac{\alpha}{1-\cot \alpha}$

Stem lodging occurs when the shoot bending moment exceeds the shoot failure moment $B_{s}$ expressed as:

$B_{S}=\frac{\sigma \pi a^{3}}{4}\left(1-\left(\frac{a-t}{a}\right)^{4}\right)$

Where $\sigma$ is the yield stress at any point along the stem, $a$ is the corresponding mean radius of the stem and $t$ is the mean stem wall thickness.

Similarly the moment acting on the root system $\left(B_{N}\right)$ is specified in Baker et al. (2014) as,

$B_{N}=\frac{0.5 \rho A C_{F} \bar{U}^{2}\left(1+\left(2 \pi f_{n}\right)^{2}(X / g)\right)}{\left(2 \pi f_{n}\right)^{2}(X / g)}\left(1+3.44 I_{u}\right) N$

Where $N$ is the number of shoots per plant.

Root lodging occurs when the wind-induced root bending moment exceeds anchorage failure moment $\left(B_{R}\right)$, which is calculated from the root plate spread $(d)$, the shear strength of the 
141 The mean wind speeds required to cause stem lodging $\left(\bar{U}_{L S}\right)$ and root lodging $\left(\bar{U}_{L R}\right)$ are

surrounding soil $(s)$ and a constant $\left(k_{4}\right)$ which is estimated by this study to have a value of 0 .73 (Eq. 5).

$$
B_{R}=s d^{3} k_{4}
$$

calculated by rearranging the bending moment expressions in Equations (1) and (4)

combining with Equations (3) and (5) respectively:

$\bar{U}_{L S}=\left[\frac{\omega_{n}^{2}\left(\frac{X}{g}\right) n\left(\frac{\sigma \pi a^{3}}{4}\right)\left(1-\left(\frac{(a-t)}{a}\right)^{4}\right)}{\left(1+\omega_{n}^{2}\left(\frac{X}{g}\right)\right)\left(0.5 \rho A C_{F} X\right)\left(\cos \left(\frac{\alpha l}{h}\right)-\cot \alpha \sin \left(\frac{\alpha l}{h}\right)\right)\left(1+6.86 I_{u}\left(1+0.366\left(\frac{\pi}{4 \theta}\right)\right)^{0.5}\right)}\right]^{0.5}$

$\bar{U}_{L R}=\left[\frac{\omega_{n}^{2}\left(\frac{X}{g}\right) \gamma S d^{3}}{\left(1+\omega_{n}^{2}\left(\frac{X}{g}\right)\right)\left(0.5 \rho A_{C F} X\right)\left(1+3.44 I_{u}\right)}\right]^{0.5}$

\section{Field experimental methods}

Field experiments involving different plant population and $\mathrm{N}$ rate treatments were set up to produce maize crops with a range of lodging risks. Measurements on these field experiments were carried out to provide data for calibrating the anchorage model, evaluating the general plausibility of the lodging model, better understand how agronomic factors affect the risk of stem and root lodging and to identify which early growing season plant characters may predict lodging risk.

\subsection{Experiments}

Field experiments were set up in the UK in 2017 (UK17), UK in 2018 (UK18) and China in 2018 (CH18). The UK experiments were conducted at ADAS Gleadthorpe near Mansfield, Nottinghamshire on a loamy sand over sandstone. The experimental design was a two-way 
factorial with plant population density $\left(4,6,12\right.$ plants $\left./ \mathrm{m}^{2}\right)$ and rate of $\mathrm{N}$ fertiliser $(0,100$ and $200 \mathrm{~kg} / \mathrm{ha} \mathrm{N}$, with $50 \%$ at sowing and $50 \%$ at leaf 4 ) as the treatment factors, and each treatment combination replicated three times. The plot size was $3 \mathrm{~m} \times 12 \mathrm{~m}$. The variety was Dualto. The crops were planted in May and harvested for forage in September. The China experiment was conducted in Lishu County (43530E), Jilin Province in Northeast China, on Black soil (USDA Haploboroll). The experimental design was a split-plot with plant population density $\left(5.5,7,8.5\right.$ and 10 plants $\left./ \mathrm{m}^{2}\right)$ as the treatment factor on the main plots and rate of $\mathrm{N}$ fertiliser rate $(0,60,120,180,240$ and $300 \mathrm{~kg} / \mathrm{ha} \mathrm{N}$, with $33 \%$ at sowing and $67 \%$ at leaf 8) as the treatment factor on the sub-plots, with each treatment combination replicated three times. The plot size was $9 \mathrm{~m}$ x $12 \mathrm{~m}$. A mid late maturing variety Liangyu 66 was used. The crops were planted in May and harvested for grain in early October.

\subsection{Measurements}

\subsubsection{Pre flowering plant characters}

The green leaf area index (LAI) was measured when leaves 4, 6 and 8 were fully emerged using a moving belt leaf area meter (Li-Cor Model 3100, Delta-T Devices, Burwell, Cambridge, UK). Plant height, fresh weight and dry weight was measured on 16 plants sampled from each plot.

\subsubsection{Post-flowering plant characters}

The field experiment was regularly inspected for any incidences of natural lodging between flowering and harvest.

\subsubsection{Shoot characteristics}

Natural Frequency was measured in the field on ten plants per plot when cobs were at the milky ripe stage. The plant was isolated from neighbouring plants and its shoot displaced by $0.20 \mathrm{~m}$ from the vertical and released. The time for three complete oscillations to occur in the 
line of displacement was recorded. Natural frequency $(\mathrm{Hz})$ was calculated as the number of oscillations divided by the timed period (seconds) (Berry et al., 2000).

184 Plants were cut off at ground level and crop height was measured from the stem base to the top of the inflorescence. The entire shoot was balanced on a pivot and its height at centre of gravity was measured as the distance from the point of balance to the base of the stem (mm).

\subsubsection{Stem characteristics}

Each internode was numbered starting with internode 1 at the bottom of the plant. The length $(\mathrm{mm})$ of each internode was measured using a ruler and the diameter $(\mathrm{mm})$ was measured at the middle of each internode using digital callipers (Etalon, Switzerland). The breaking strength (Newtons) of internodes 1-3 (combined), internode 5 and internode 8 were measured using a three-point bending test with a digital Force Gauge (Mecmesin Ltd, Horsham, UK) width were recorded at right angles to each other (Figure 1).

A

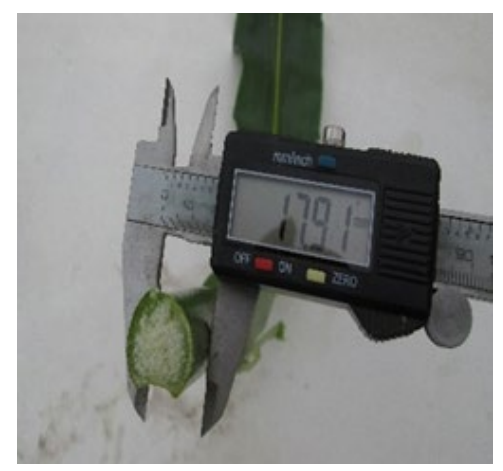

$\mathrm{B}$

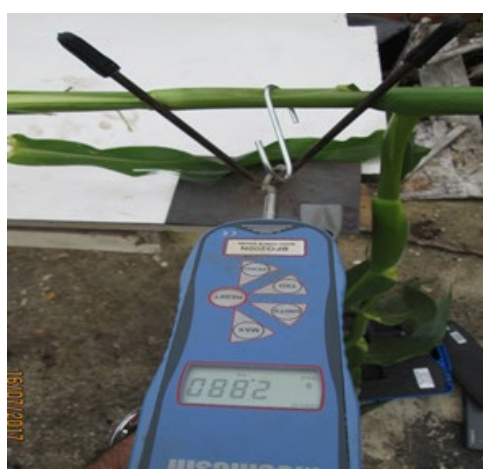

$\mathrm{C}$

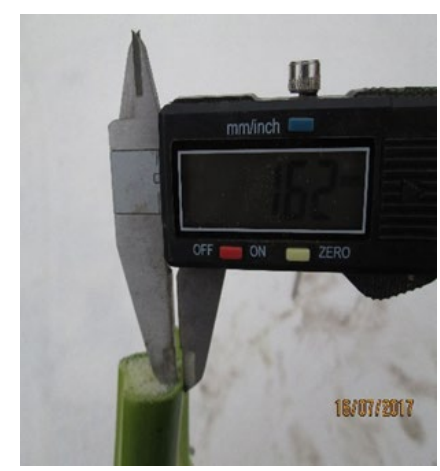

Figure 1. A) measurement of stem diameter, B) three-point breaking test used stem for stem breaking strength determination, C) measurement of stem wall width

\subsubsection{Root characteristics}


198 Ten plants per plot were excavated to a soil depth of about $0.20 \mathrm{~m}$ taking care not to break off 199 the brace roots. The soil was carefully removed from the roots and the brace roots identified 200 as those that emerge above the soil surface and the crown roots as those that emerge from 201 below the soil surface (Figure 2). The number of brace roots and the number of crown roots 202 were counted on each plant. The maximum crown root plate spread at a soil depth of $80 \mathrm{~mm}$ was measured along with the crown root plate spread at $90^{\circ}$ to the maximum crown root spread and the maximum depth of roots that were extracted (Figure 2). For the brace roots, the maximum root plate spread at soil surface, the root plate spread at $90^{\circ}$ to the maximum root spread and the maximum height above the soil surface where they joined the stem was recorded (Figure 2).

208 At the cob milky ripe stage, root anchorage strength was measured on a separate set of experimental plots to the main experiment described above, with plant population densities of 4, 6 and 12 plants $/ \mathrm{m}^{2}$. These measurements were made using a Mecmesin force gauge by applying a perpendicular force to the stem at $0.10 \mathrm{~m}$ from the ground (Fouere et al., 1995; Shengqun et al., 2012). The maximum force (Newtons) to displace the stem by 45 degrees

213 from the vertical was recorded, together with the soil shear strength at $0.10 \mathrm{~m}$ soil depth using 214 a shear vane (Pilcon, Basingstoke, UK). 


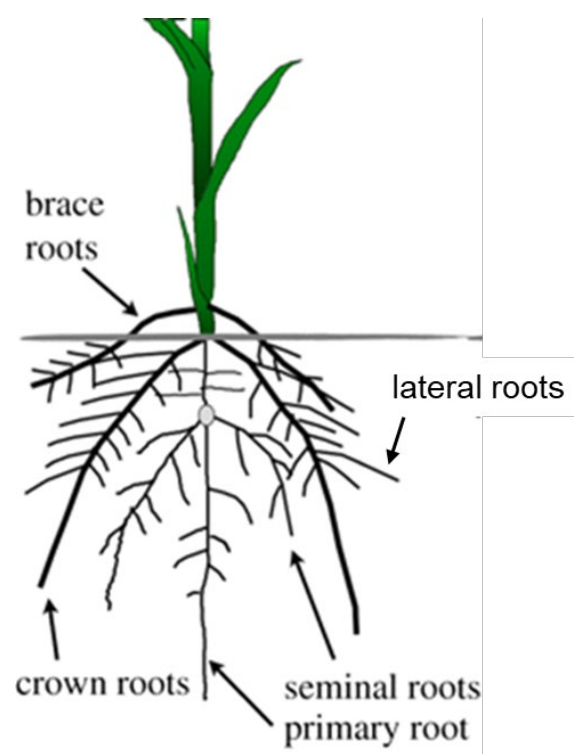

216 Figure 2 Root structure of maize

The stem failure moment $\left(B_{S}\right)$ was calculated from the measured tensile failure strength $\left(F_{S}\right)$

221 and length $(L)$ of the internode, as described in Berry et al. (2000).

222

$$
B_{S}=\frac{F_{S} L}{4}
$$

224 Anchorage failure moment $\left(B_{R}\right)$ was calculated according to equation 5 .

\subsection{Statistical analysis}

227 Analysis of variance procedures for fully randomised two-way factorial and split-plot experimental designs were used within Genstat 18 software to calculate standard errors of differences between means (S.E.D.) and significant differences between treatments. Genstat 
was also used to perform linear regression analysis and calculate correlation coefficients between the measured plant characteristics.

\section{Results}

\subsection{Anchorage strength}

235 The anchorage strength tests showed that reducing plant population from 12 to 4 plants $/ \mathrm{m}^{2}$ significantly increased anchorage failure moment from $23.7 \mathrm{Nm}$ to $45.5 \mathrm{Nm}$, average crown root spread from $158 \mathrm{~mm}$ to $208 \mathrm{~mm}$, crown root number per plant from 11.6 to 14.0 , brace root number from 0.6 to 10.2 per plant and root fresh weight from $180 \mathrm{~g}$ to $580 \mathrm{~g}(\mathrm{P}<0.001)$.

239 Linear regression analysis was carried out to test whether the anchorage failure moment was

240 linearly related to the product of the crown root plate spread and shear strength of the surrounding soil, as has been found for cereal species. This showed a significant positive

242 relationship $(\mathrm{P}<0.01$; Figure 3$)$ with a slope of 0.073 and intercept of $16.7 \mathrm{Nm}$. This

243 relationship was used to calibrate the lodging model's calculation of anchorage failure

244 moment (Equation 5). Multiplying the product of crown root plate spread cubed and soil

245 shear strength by crown root number increased the $\mathrm{R}^{2}$ value by a modest amount from 0.54 to

246 0.60. Of the root parameters measured, root fresh weight had the strongest relationship with 247 the anchorage failure moment with an $\mathrm{R}^{2}$ of 0.80 . 


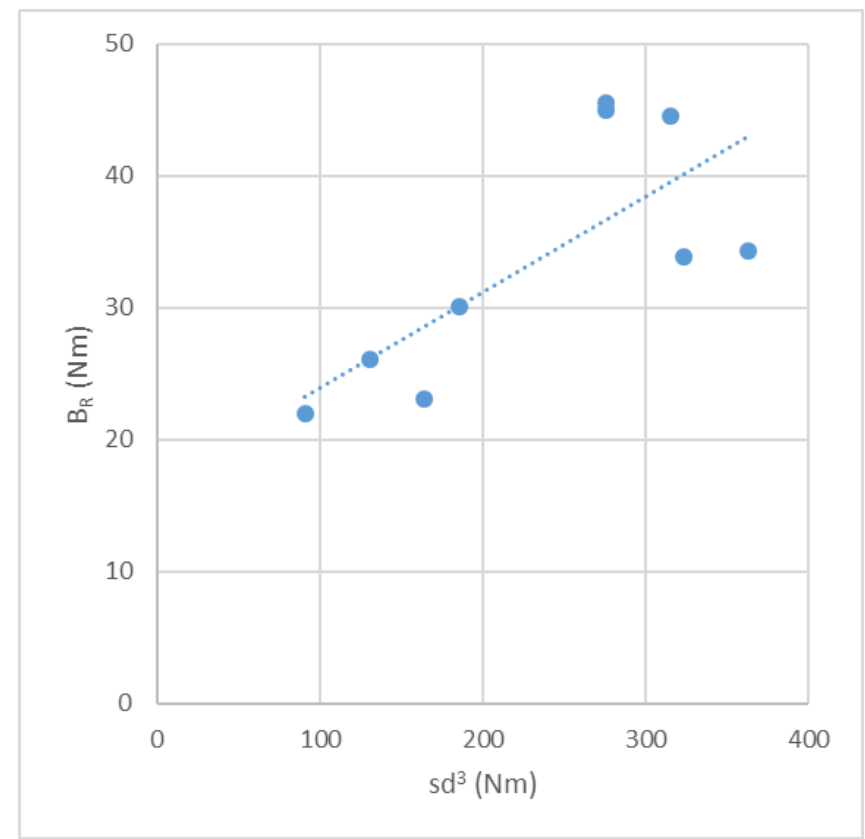

Figure 3. Relationship between measured anchorage failure moment (BR) and the product of crown root plate (d) cubed and soil strength (s), $\left.\mathrm{y}=0.073 \mathrm{x}+16.7 ; \mathrm{R}^{2}=0.54 ; \mathrm{P}<0.01\right)$.

The effects of plant population and $\mathrm{N}$ fertiliser rate on the measurements of the plant characters associated with lodging are described in tables 1 to 6 , with effects on the calculations of stem failure wind speed described in Tables 7 and 8). Increasing plant population from 4 to 12 plants $/ \mathrm{m}^{2}$ increased the biomass yield from 16.7 to $25.5 \mathrm{t} /$ ha for the UK17 experiment and from $12.1 \mathrm{t} /$ ha to $15.6 \mathrm{t} / \mathrm{ha}$ in the UK18 experiment $(\mathrm{P}<0.001$; Tables 1 and 2). Increasing $\mathrm{N}$ rate did not significantly $(\mathrm{P}>0.05)$ increase biomass in the UK experiments. By contrast, in the $\mathrm{CH} 18$ experiment the plant population and $\mathrm{N}$ rate treatments interacted such that increasing plant population from 5.5 to $10 \mathrm{plants} / \mathrm{m}^{2}$ increased biomass yield at low $\mathrm{N}$ rates only and had no effect when fertilised at 180 or $300 \mathrm{~kg} \mathrm{~N} / \mathrm{ha}$. Increasing $\mathrm{N}$ rate significantly $(\mathrm{P}<0.05)$ increased biomass at all plant populations, except $10 \mathrm{plants} / \mathrm{m}^{2}$ (Table 3). 
Increasing the plant population significantly $(\mathrm{P}<0.01)$ reduced the anchorage and stem failure wind speeds in all experiments (Tables 7 and 8), thus indicating greater risk to root and stem lodging. Increasing the plant population increased the risk of lodging because it significantly reduced the spread of the root plate in all experiments and the depth of the root plate in the UK17 and UK18 experiments (Table 1, 2 and 3). Increasing plant population also increased the leverage exerted on the plant base by increasing plant height and height at centre of gravity in the UK17 and CH18 experiments and reducing the plant's natural frequency (rate of shoot oscillation) in all experiments (Table 1, 2 and 3). Increasing plant population increased the risk of stem lodging by reducing stem strength as a result of narrower stems in all experiments and additionally as a result of thinner walled stems in the UK17 and UK18 experiments (Table 4, 5 and 6).

Increasing the rate of $\mathrm{N}$ fertiliser did not significantly affect the stem failure wind speeds in any of the experiments (Tables $7 \& 8$ ). In the UK17 and UK18 experiments, this was because changes in $\mathrm{N}$ rate did not significantly affect the plant characteristics that determine plant leverage or stem strength. In the CH18 experiment, the effect of increasing plant height with greater $\mathrm{N}$ on stem failure windspeed was counteracted by an increase in strength of internodes 5 and 8 as $\mathrm{N}$ fertiliser rate was increased from 60 to $300 \mathrm{~kg} \mathrm{~N} / \mathrm{ha}$. However, increasing $\mathrm{N}$ rate did reduce the root failure wind speed in $\mathrm{CH} 18(\mathrm{P}<0.01$; Table 8$)$, primarily as a result of its effect to increase leverage.

Table 1. UK17: Biomass yield and character associated with plant leverage and anchorage strength

\begin{tabular}{|c|l|l|l|l|l|l|l|}
\hline Plants $/ \mathrm{m}^{2}$ & $\begin{array}{l}\mathrm{N} \\
\text { applied } \\
\mathrm{kg} / \mathrm{ha}\end{array}$ & $\begin{array}{l}\text { Biomass } \\
\text { yield at } \\
\text { harvest } \\
(\mathrm{t} / \mathrm{ha})\end{array}$ & $\begin{array}{l}\text { Plant } \\
\text { height } \\
(\mathrm{cm})\end{array}$ & $\begin{array}{l}\text { Height at } \\
\text { centre of } \\
\text { gravity } \\
(\mathrm{cm})\end{array}$ & $\begin{array}{l}\text { Natural } \\
\text { Frequency } \\
(\mathrm{Hz})\end{array}$ & $\begin{array}{l}\text { Root plate } \\
\text { spread } \\
(\mathrm{cm})\end{array}$ & $\begin{array}{l}\text { Root } \\
\text { plate } \\
\text { depth } \\
(\mathrm{cm})\end{array}$ \\
\hline 4 & 0 & 17.6 & 246 & 85.7 & 1.22 & 16.0 & 10.74 \\
\hline 4 & 100 & 16.2 & 241 & 85.4 & 1.16 & 16.2 & 10.32 \\
\hline
\end{tabular}




\begin{tabular}{|c|c|c|c|c|c|c|c|}
\hline 4 & 200 & 16.3 & 241 & 86.5 & 1.15 & 17.3 & 10.72 \\
\hline 6 & 0 & 20.5 & 257 & 93.1 & 1.07 & 16.1 & 11.03 \\
\hline 6 & 100 & 20.2 & 256 & 93.6 & 0.97 & 15.3 & 9.77 \\
\hline 6 & 200 & 19.8 & 258 & 93.2 & 0.99 & 15.2 & 9.89 \\
\hline 12 & 0 & 25.7 & 285 & 109.2 & 0.81 & 12.1 & 8.91 \\
\hline 12 & 100 & 26.4 & 280 & 106.5 & 0.72 & 13.1 & 8.82 \\
\hline 12 & 200 & 24.4 & 276 & 108.9 & 0.76 & 13.0 & 8.85 \\
\hline 4 & mean & 16.7 & 243 & 85.9 & 1.177 & 16.5 & 10.59 \\
\hline 6 & mean & 20.2 & 257 & 93.3 & 1.010 & 15.5 & 10.23 \\
\hline 12 & mean & 25.5 & 280 & 108.2 & 0.763 & 12.7 & 8.86 \\
\hline mean & 0 & 21.2 & 263 & 96.0 & 1.033 & 14.7 & 10.23 \\
\hline mean & 100 & 20.9 & 259 & 95.2 & 0.950 & 14.9 & 9.64 \\
\hline mean & 200 & 20.2 & 258 & 96.2 & 0.967 & 15.2 & 9.82 \\
\hline Plants $/ \mathrm{m}^{2}$ & SED & $0.540 * * *$ & $2.7 * * *$ & $0.58 * * *$ & $0.038^{* * *}$ & $0.47 * * *$ & $0.890 * *$ \\
\hline Nitrogen & SED & 0.540 & 2.7 & 0.58 & 0.038 & 0.47 & 0.890 \\
\hline Interaction & SED & 0.936 & 4.6 & 1.01 & 0.138 & 0.82 & 1.541 \\
\hline
\end{tabular}

285 Table 2. UK18: Biomass yield and character associated with plant leverage and anchorage 286 strength.

\begin{tabular}{|c|c|c|c|c|c|c|c|}
\hline Plants $/ \mathrm{m}^{2}$ & $\begin{array}{l}\mathrm{N} \text { rate } \\
\mathrm{kg} / \mathrm{ha}\end{array}$ & $\begin{array}{l}\text { Biomass } \\
\text { yield at } \\
\text { harvest } \\
(\mathrm{t} / \mathrm{ha})\end{array}$ & $\begin{array}{l}\text { Plant } \\
\text { height } \\
(\mathrm{cm})\end{array}$ & $\begin{array}{l}\text { Height to } \\
\text { centre of } \\
\text { gravity } \\
(\mathrm{cm})\end{array}$ & $\begin{array}{l}\text { Natural } \\
\text { Frequency } \\
(\mathrm{Hz})\end{array}$ & $\begin{array}{l}\text { Root plate } \\
\text { spread } \\
(\mathrm{cm})\end{array}$ & $\begin{array}{l}\text { Root } \\
\text { plate } \\
\text { depth } \\
(\mathrm{cm})\end{array}$ \\
\hline 4 & 0 & 13.7 & 206 & 74.5 & 1.19 & 20.8 & 8.80 \\
\hline 4 & 100 & 10.6 & 197 & 69.3 & 1.08 & 21.9 & 9.29 \\
\hline 4 & 200 & 11.9 & 202 & 73.4 & 1.11 & 20.4 & 8.75 \\
\hline 6 & 0 & 12.1 & 197 & 71.2 & 1.19 & 20.0 & 8.59 \\
\hline 6 & 100 & 12.5 & 205 & 76.2 & 1.14 & 20.7 & 8.06 \\
\hline 6 & 200 & 14.1 & 201 & 76.2 & 1.03 & 18.4 & 8.33 \\
\hline 12 & 0 & 15.8 & 212 & 81.4 & 1.04 & 16.6 & 7.24 \\
\hline 12 & 100 & 14.8 & 195 & 70.2 & 1.03 & 13.7 & 6.88 \\
\hline 12 & 200 & 16.2 & 198 & 72.8 & 0.99 & 15.8 & 7.08 \\
\hline 4 & mean & 12.1 & 202 & 72.4 & 1.13 & 21.1 & 8.95 \\
\hline 6 & mean & 12.9 & 201 & 74.5 & 1.12 & 19.7 & 8.33 \\
\hline 12 & mean & 15.6 & 202 & 74.8 & 1.02 & 15.3 & 7.07 \\
\hline mean & 0 & 13.9 & 205 & 75.7 & 1.14 & 19.1 & 8.21 \\
\hline mean & 100 & 12.6 & 199 & 71.9 & 1.08 & 18.8 & 8.08 \\
\hline mean & 200 & 14.1 & 200 & 74.1 & 1.04 & 18.2 & 8.05 \\
\hline Plants $/ \mathrm{m}^{2}$ & SED & $1.568 * *$ & 6.06 & 2.426 & $0.0357 *$ & $1.009 * * *$ & $0.441^{* *}$ \\
\hline Nitrogen & SED & 1.568 & 6.06 & 2.426 & 0.0357 & 1.009 & 0.441 \\
\hline Interaction & SED & 2.716 & 10.5 & 4.202 & 0.0618 & 1.748 & 0.736 \\
\hline
\end{tabular}


Table 3. CH18: Biomass yield and characters associated with plant leverage and anchorage strength

\begin{tabular}{|c|c|c|c|c|c|c|c|}
\hline Plants/m² & $\begin{array}{l}\mathrm{N} \text { rate } \\
\mathrm{kg} / \mathrm{ha}\end{array}$ & $\begin{array}{l}\text { Biomas } \\
\mathrm{s} \text { at } \\
\text { harvest } \\
(\mathrm{t} / \mathrm{ha})\end{array}$ & $\begin{array}{l}\text { Total } \\
\text { plant } \\
\text { height } \\
(\mathrm{cm})\end{array}$ & $\begin{array}{l}\text { Height to } \\
\text { centre of } \\
\text { gravity } \\
(\mathrm{cm})\end{array}$ & $\begin{array}{l}\text { Natural } \\
\text { Frequenc } \\
\mathrm{y} \\
(\mathrm{Hz})\end{array}$ & $\begin{array}{l}\text { Root plate } \\
\text { spread } \\
(\mathrm{cm})\end{array}$ & $\begin{array}{l}\text { Root plate } \\
\text { depth } \\
(\mathrm{cm})\end{array}$ \\
\hline 5.5 & 60 & 17.0 & 232 & 87.5 & 0.882 & 14.9 & 10.47 \\
\hline 5.5 & 180 & 21.5 & 261 & 98.1 & 0.831 & 16.2 & 10.82 \\
\hline 5.5 & 300 & 23.8 & 270 & 103.7 & 0.823 & 17.4 & 11.03 \\
\hline 7 & 60 & 15.2 & 231 & 87.9 & 0.868 & 13.9 & 10.50 \\
\hline 7 & 180 & 24.5 & 259 & 101.2 & 0.815 & 15.1 & 8.93 \\
\hline 7 & 300 & 25.5 & 271 & 106.1 & 0.749 & 16.1 & 9.77 \\
\hline 8.5 & 60 & 16.4 & 226 & 85.7 & 0.806 & 14.7 & 9.73 \\
\hline 8.5 & 180 & 26.9 & 264 & 103.5 & 0.775 & 14.7 & 9.32 \\
\hline 8.5 & 300 & 25.2 & 268 & 107.2 & 0.757 & 15.3 & 9.56 \\
\hline 10 & 60 & 23.1 & 244 & 93.6 & 0.759 & 14.0 & 9.14 \\
\hline 10 & 180 & 23.0 & 263 & 103.2 & 0.773 & 13.2 & 9.37 \\
\hline 10 & 300 & 25.6 & 267 & 105.8 & 0.719 & 14.5 & 10.62 \\
\hline 5.5 & mean & 20.8 & 254 & 96.4 & 0.845 & 16.1 & 10.77 \\
\hline 7 & mean & 21.7 & 254 & 98.4 & 0.811 & 15.0 & 9.73 \\
\hline 8.5 & mean & 22.8 & 253 & 98.8 & 0.779 & 14.9 & 9.54 \\
\hline 10 & mean & 23.9 & 258 & 100.9 & 0.750 & 13.9 & 9.71 \\
\hline mean & 60 & 17.9 & 234 & 88.7 & 0.829 & 14.4 & 9.96 \\
\hline mean & 180 & 24.0 & 262 & 101.5 & 0.799 & 14.8 & 9.61 \\
\hline mean & 300 & 25.0 & 269 & 105.7 & 0.762 & 15.8 & 10.24 \\
\hline Plants $/ \mathrm{m}^{2}$ & SED & 1.67 & 3.9 & $1.12 *$ & $0.016 * *$ & $0.49 *$ & 0.88 \\
\hline Nitrogen & SED & $\begin{array}{l}1.02 * * \\
*\end{array}$ & $\begin{array}{l}3.2^{* *} \\
*\end{array}$ & $1.49 * * *$ & $0.015^{* * *}$ & $0.33 * *$ & 0.42 \\
\hline Interaction & SED & $2.36^{*}$ & 6.5 & 2.69 & 0.029 & 0.72 & 1.11 \\
\hline
\end{tabular}


Table 4. UK17: Length, diameter, wall width and failure moment for the bottom three internodes (I/N 1-3), internode 2 (I/N 2), internode 5 (I/N $2925)$ and internode 8 (I/N 8).

\begin{tabular}{|c|c|c|c|c|c|c|c|c|c|c|c|c|c|}
\hline \multirow[t]{2}{*}{ Plants/m2 } & \multirow{2}{*}{$\begin{array}{l}\mathrm{N} \\
\text { rate } \\
\mathrm{kg} / \mathrm{ha}\end{array}$} & \multicolumn{3}{|c|}{ Internode Length (cm) } & \multicolumn{3}{|c|}{ Internode Diameter (mm) } & \multicolumn{3}{|c|}{ Wall width (mm) } & \multicolumn{3}{|c|}{ Stem Failure moment (Nm) } \\
\hline & & $\mathrm{I} / \mathrm{N} 1-3$ & $\mathrm{I} / \mathrm{N} 5$ & $\begin{array}{l}\mathrm{I} / \mathrm{N} \\
8\end{array}$ & $\mathrm{I} / \mathrm{N} 2$ & $\mathrm{I} / \mathrm{N} 5$ & $\mathrm{I} / \mathrm{N} 8$ & $\mathrm{I} / \mathrm{N} 2$ & $\mathrm{I} / \mathrm{N} 5$ & $\mathrm{I} / \mathrm{N} 8$ & $\mathrm{I} / \mathrm{N} 1-3$ & $\mathrm{I} / \mathrm{N} 5$ & $\mathrm{I} / \mathrm{N} 8$ \\
\hline 4 & 0 & 31.8 & 21.8 & 22.5 & 27.1 & 21.8 & 18.3 & 3.23 & 1.34 & 0.759 & 19.0 & 7.75 & 4.74 \\
\hline 4 & 100 & 39.4 & 21.3 & 22.0 & 27.4 & 20.7 & 18.3 & 3.02 & 1.47 & 0.713 & 19.1 & 7.44 & 4.69 \\
\hline 4 & 200 & 32.5 & 21.6 & 21.2 & 26.8 & 21.1 & 17.6 & 2.71 & 1.24 & 0.691 & 16.9 & 7.10 & 4.19 \\
\hline 6 & 0 & 37.4 & 24.0 & 22.7 & 25.4 & 19.6 & 17.0 & 2.83 & 1.40 & 0.699 & 18.3 & 6.54 & 3.53 \\
\hline 6 & 100 & 34.1 & 23.5 & 22.2 & 25.8 & 20.9 & 18.0 & 2.84 & 1.34 & 0.790 & 16.7 & 7.06 & 3.80 \\
\hline 6 & 200 & 33.8 & 23.4 & 22.9 & 25.7 & 20.7 & 17.9 & 2.68 & 1.32 & 0.728 & 17.5 & 7.25 & 3.53 \\
\hline 12 & 0 & 48.8 & 25.9 & 22.4 & 21.0 & 18.7 & 14.7 & 1.92 & 1.05 & 0.777 & 11.5 & 4.42 & 2.35 \\
\hline 12 & 100 & 49.1 & 26.2 & 22.3 & 21.2 & 18.3 & 14.7 & 1.89 & 1.08 & 0.710 & 12.7 & 4.39 & 2.34 \\
\hline 12 & 200 & 44.7 & 26.0 & 22.4 & 21.4 & 18.6 & 15.2 & 1.94 & 0.90 & 0.650 & 11.5 & 4.36 & 2.84 \\
\hline 4 & mean & 34.6 & 21.6 & 21.9 & 27.1 & 21.2 & 18.1 & 2.99 & 1.35 & 0.721 & 18.3 & 7.43 & 4.54 \\
\hline 6 & mean & 35.1 & 23.6 & 22.6 & 25.6 & 20.4 & 17.6 & 2.78 & 1.35 & 0.739 & 17.5 & 6.95 & 3.62 \\
\hline 12 & mean & 47.5 & 26.0 & 22.4 & 21.2 & 18.5 & 14.9 & 1.92 & 1.01 & 0.712 & 11.9 & 4.39 & 2.51 \\
\hline mean & 0 & 39.3 & 23.9 & 22.5 & 24.5 & 20.0 & 16.7 & 2.66 & 1.26 & 0.745 & 16.2 & 6.24 & 3.54 \\
\hline mean & 100 & 40.9 & 23.7 & 22.2 & 24.8 & 20.0 & 17.0 & 2.58 & 1.30 & 0.738 & 16.2 & 6.30 & 3.61 \\
\hline mean & 200 & 37.0 & 23.7 & 22.2 & 24.6 & 20.1 & 16.9 & 2.44 & 1.15 & 0.690 & 15.3 & 6.24 & 3.52 \\
\hline Plants/m2 & SED & $2.64 * * *$ & $0.49 * * *$ & 0.64 & $0.30 * * *$ & $0.40 * * *$ & $0.23 * * *$ & $0.166^{* * *}$ & 0.056 & $0.074 * * *$ & $1.43 * * *$ & $0.243 * * *$ & $0.224 * * *$ \\
\hline Nitrogen & SED & 2.64 & 0.49 & 0.64 & 0.30 & 0.4 & 0.23 & 0.166 & 0.056 & 0.074 & 1.43 & 0.243 & 0.224 \\
\hline Interaction & SED & 4.58 & 0.849 & 1.11 & 0.52 & 0.70 & 0.40 & 0.269 & 0.098 & 0.123 & 2.48 & 0.422 & 0.389 \\
\hline
\end{tabular}


Table 5. UK18: Length, diameter, wall width and failure moment for the bottom three internodes (I/N 1-3), internode 2 (I/N 2), internode 5 (I/N 5) and internode 8 (I/N 8).

\begin{tabular}{|c|c|c|c|c|c|c|c|c|c|c|c|c|c|}
\hline \multirow[t]{2}{*}{ Plants $/ \mathrm{m}^{2}$} & \multirow[t]{2}{*}{$\begin{array}{l}\mathrm{N} \text { rate } \\
\mathrm{kg} / \mathrm{ha}\end{array}$} & \multicolumn{3}{|c|}{$\begin{array}{l}\text { Internode Length } \\
(\mathrm{cm})\end{array}$} & \multicolumn{3}{|c|}{ Internode Diameter (mm) } & \multicolumn{3}{|c|}{ Wall width (mm) } & \multicolumn{3}{|c|}{ Stem Failure moment (Nm) } \\
\hline & & $\begin{array}{l}\text { I/N 1- } \\
3 \\
\end{array}$ & $\begin{array}{l}\mathrm{I} / \mathrm{N} \\
5 \\
\end{array}$ & $\mathrm{I} / \mathrm{N} 8$ & $\mathrm{I} / \mathrm{N} 2$ & $\mathrm{I} / \mathrm{N} 5$ & $\mathrm{I} / \mathrm{N} 8$ & $\mathrm{I} / \mathrm{N} 2$ & $\mathrm{I} / \mathrm{N} 5$ & $\mathrm{I} / \mathrm{N} 8$ & $\mathrm{I} / \mathrm{N} 1-3$ & $\mathrm{I} / \mathrm{N} 5$ & $\mathrm{I} / \mathrm{N} 8$ \\
\hline 4 & 0 & 29.8 & 15.5 & 13.8 & 24.7 & 21.0 & 12.96 & 1.095 & 0.781 & 0.498 & 10.55 & 4.52 & 1.96 \\
\hline 4 & 100 & 26.8 & 14.6 & 13.0 & 23.6 & 18.5 & 12.88 & 1.049 & 0.738 & 0.482 & 8.46 & 3.74 & 2.06 \\
\hline 4 & 200 & 30.6 & 15.0 & 13.8 & 23.3 & 18.3 & 11.84 & 0.912 & 0.775 & 0.480 & 9.74 & 3.96 & 1.81 \\
\hline 6 & 0 & 28.5 & 14.6 & 20.2 & 22.0 & 17.3 & 11.86 & 0.875 & 0.750 & 0.498 & 8.26 & 3.40 & 1.51 \\
\hline 6 & 100 & 29.1 & 16.4 & 13.1 & 22.2 & 18.2 & 11.91 & 0.941 & 0.702 & 0.443 & 7.84 & 3.54 & 1.53 \\
\hline 6 & 200 & 27.3 & 17.0 & 13.1 & 21.0 & 18.3 & 11.62 & 0.837 & 0.671 & 0.431 & 7.42 & 2.04 & 1.30 \\
\hline 12 & 0 & 33.4 & 18.2 & 13.4 & 19.4 & 16.3 & 10.40 & 0.816 & 0.537 & 0.415 & 6.92 & 3.97 & 1.10 \\
\hline 12 & 100 & 31.8 & 15.3 & 12.6 & 19.5 & 15.1 & 9.89 & 0.776 & 0.500 & 0.375 & 5.99 & 3.13 & 0.89 \\
\hline 12 & 200 & 29.4 & 16.7 & 12.8 & 19.5 & 15.1 & 10.05 & 0.699 & 0.540 & 0.354 & 5.71 & 2.16 & 0.85 \\
\hline 4 & mean & 29.1 & 15.0 & 13.5 & 23.9 & 19.2 & 12.56 & 1.019 & 0.765 & 0.487 & 9.58 & \begin{tabular}{|l|}
4.07 \\
\end{tabular} & 1.94 \\
\hline 6 & mean & 28.3 & 16.0 & 15.5 & 21.8 & 17.9 & 11.80 & 0.884 & 0.708 & 0.457 & 7.84 & 2.99 & 1.45 \\
\hline 12 & mean & 31.5 & 16.7 & 12.9 & 19.5 & 15.5 & 10.11 & 0.764 & 0.526 & 0.381 & 6.21 & 3.09 & 0.95 \\
\hline mean & 0 & 30.6 & 16.1 & 15.8 & 22.0 & 18.2 & 11.74 & 0.929 & 0.689 & 0.470 & 8.58 & 3.96 & 1.52 \\
\hline mean & 100 & 29.2 & 15.4 & 12.9 & 21.8 & 17.3 & 11.56 & 0.922 & 0.647 & 0.433 & 7.43 & 3.47 & 1.49 \\
\hline mean & 200 & 29.1 & 16.2 & 13.2 & 21.3 & 17.2 & 11.17 & 0.816 & 0.662 & 0.422 & 7.62 & 2.72 & 1.32 \\
\hline Plants $/ \mathrm{m}^{2}$ & SED & 1.31 & 0.80 & 1.86 & $0.62 * * *$ & $0.81 * * *$ & $0.335^{* * *}$ & $0.0463 * * *$ & $0.0384 * *$ & 0.236 & $0.656^{* * *}$ & $0.243^{* * *}$ & $0.103^{*} * *$ \\
\hline Nitrogen & SED & 1.31 & 0.80 & 1.86 & 0.62 & 0.81 & 0.335 & $0.0463^{*}$ & 0.0384 & 0.427 & 0.656 & 0.243 & 0.103 \\
\hline Interaction & SED & 2.27 & 1.39 & 3.22 & 1.08 & 1.41 & 0.580 & 0.0801 & 0.0665 & 0.441 & 1.136 & 0.421 & 0.178 \\
\hline
\end{tabular}


Table 6. CH18: Length, diameter, wall width and failure moment for the bottom three internodes (I/N 1-3), internode 2 (I/N 2), internode 5 (I/N 5) and internode $8(\mathrm{I} / \mathrm{N} 8)$.

\begin{tabular}{|c|c|c|c|c|c|c|c|c|c|c|c|c|c|}
\hline \multirow[t]{2}{*}{ Plants $/ \mathrm{m}^{2}$} & \multirow{2}{*}{$\begin{array}{l}\mathrm{N} \text { rate } \\
\mathrm{kg} / \mathrm{ha}\end{array}$} & \multicolumn{3}{|c|}{ Internode Length (cm) } & \multicolumn{3}{|c|}{ Internode Diameter (mm) } & \multicolumn{3}{|c|}{ Wall width (mm) } & \multicolumn{3}{|c|}{ Stem Failure moment (Nm) } \\
\hline & & $\mathrm{I} / \mathrm{N} 1-3$ & $\mathrm{I} / \mathrm{N} 5$ & $\mathrm{I} / \mathrm{N} 8$ & $\mathrm{I} / \mathrm{N} 2$ & $\mathrm{I} / \mathrm{N} 5$ & $\mathrm{I} / \mathrm{N} 8$ & $\mathrm{I} / \mathrm{N} 2$ & $\mathrm{I} / \mathrm{N} 5$ & $\mathrm{I} / \mathrm{N} 8$ & $\begin{array}{l}\mathrm{I} / \mathrm{N} 1- \\
3\end{array}$ & $\mathrm{I} / \mathrm{N} 5$ & $\mathrm{I} / \mathrm{N} 8$ \\
\hline 5.5 & 60 & 24.2 & 16.4 & 13.5 & 22.4 & 21.0 & 19.5 & 1.38 & 1.53 & 1.02 & 21.4 & 9.49 & 4.47 \\
\hline 5.5 & 180 & 23.9 & 19.0 & 15.2 & 23.1 & 22.1 & 21.5 & 1.64 & 1.53 & 1.08 & 23.8 & 12.47 & 5.45 \\
\hline 5.5 & 300 & 26.1 & 19.6 & 14.8 & 25.1 & 23.6 & 22.9 & 1.74 & 1.53 & 1.36 & 27.4 & 14.09 & 7.42 \\
\hline 7 & 60 & 24.2 & 16.7 & 14.0 & 20.6 & 19.2 & 20.1 & 1.46 & 1.35 & 1.10 & 23.2 & 8.42 & 4.25 \\
\hline 7 & 180 & 26.3 & 19.3 & 15.7 & 22.7 & 21.6 & 23.4 & 1.73 & 1.51 & 1.27 & 25.0 & 11.51 & 6.13 \\
\hline 7 & 300 & 28.3 & 19.2 & 16.7 & 24.2 & 21.7 & 19.7 & 1.55 & 1.59 & 1.27 & 27.1 & 10.84 & 6.40 \\
\hline 8.5 & 60 & 23.9 & 16.2 & 13.5 & 20.3 & 19.5 & 18.8 & 1.45 & 1.44 & 1.11 & 22.0 & 7.51 & 3.59 \\
\hline 8.5 & 180 & 30.6 & 19.3 & 15.0 & 22.1 & 20.6 & 21.8 & 1.60 & 1.42 & 1.14 & 26.0 & 9.16 & 4.76 \\
\hline 8.5 & 300 & 29.0 & 19.8 & 17.2 & 23.2 & 22.3 & 20.9 & 1.56 & 1.63 & 1.37 & 23.9 & 8.74 & 4.87 \\
\hline 10 & 60 & 26.8 & 18.3 & 13.7 & 19.0 & 18.2 & 19.5 & 1.23 & 1.31 & 1.07 & 20.7 & 8.03 & 4.12 \\
\hline 10 & 180 & 29.0 & 19.0 & 15.9 & 20.3 & 19.6 & 19.8 & 1.56 & 1.33 & 1.25 & 20.2 & 7.25 & 4.24 \\
\hline 10 & 300 & 28.0 & 17.7 & 17.0 & 21.3 & 20.6 & 18.6 & 1.42 & 1.39 & 1.24 & 19.4 & 7.04 & 4.31 \\
\hline & & & & & & & & & & & & & \\
\hline 5.5 & mean & 24.7 & 18.3 & 14.5 & 23.5 & 22.2 & 21.3 & 1.59 & 1.53 & 1.15 & 24.2 & 12.02 & 5.78 \\
\hline 7 & mean & 26.3 & 18.4 & 15.5 & 22.5 & 20.8 & 21.1 & 1.58 & 1.48 & 1.22 & 25.1 & 10.26 & 5.59 \\
\hline 8.5 & mean & 27.8 & 18.5 & 15.2 & 21.9 & 20.8 & 20.5 & 1.54 & 1.50 & 1.21 & 24.0 & 8.47 & 4.41 \\
\hline 10 & mean & 28.0 & 18.3 & 15.5 & 20.2 & 19.5 & 19.3 & 1.40 & 1.34 & 1.19 & 20.1 & 7.44 & 4.22 \\
\hline mean & 60 & 24.8 & 16.9 & 13.7 & 20.6 & 19.5 & 19.5 & 1.38 & 1.41 & 1.08 & 21.9 & 8.37 & 4.11 \\
\hline mean & 180 & 27.4 & 19.2 & 15.5 & 22.1 & 21.0 & 21.6 & 1.63 & 1.45 & 1.19 & 23.7 & 10.10 & 5.14 \\
\hline mean & 300 & 27.8 & 19.1 & 16.4 & 23.5 & 22.0 & 20.5 & 1.57 & 1.53 & 1.31 & 24.5 & 10.18 & 5.75 \\
\hline Plants $/ \mathrm{m}^{2}$ & SED & 1.47 & 0.76 & 0.60 & $0.52 * *$ & $0.49 * *$ & 1.34 & 0.147 & 0.098 & 0.074 & 1.48 & $1.173^{*}$ & $0.541 * *$ \\
\hline Nitrogen & SED & $0.92 * *$ & $0.34 * * *$ & $0.39 * * *$ & $0.31 * * *$ & $0.37 * * *$ & $0.63 *$ & $0.052^{* * *}$ & 0.062 & $0.051^{* *}$ & 1.53 & $0.556^{* *}$ & $0.347 * * *$ \\
\hline Interaction & SED & 2.10 & $0.94 *$ & 0.87 & 0.73 & 0.77 & 1.69 & 0.170 & 0.140 & 0.111 & 2.90 & 1.483 & 0.783 \\
\hline
\end{tabular}


Table 7. UK17 and UK18: Stem and anchorage failure wind speeds for the bottom three internodes (I/N 1-3), internode 2 (I/N 2), internode 5 (I/N 5) and internode 8 (I/N 8).

\begin{tabular}{|c|c|c|c|c|c|c|c|c|c|}
\hline & & \multicolumn{4}{|c|}{ UK17 } & \multicolumn{4}{|c|}{ UK18 } \\
\hline Plants $/ \mathrm{m}^{2}$ & $\begin{array}{l}\mathrm{N} \\
\text { applied } \\
\mathrm{kg} / \mathrm{ha}\end{array}$ & $\begin{array}{l}\text { Anchorage } \\
(\mathrm{m} / \mathrm{s})\end{array}$ & $\begin{array}{l}\mathrm{I} / \mathrm{N} 1-3 \\
(\mathrm{~m} / \mathrm{s})\end{array}$ & $\begin{array}{l}\mathrm{I} / \mathrm{N} 5 \\
(\mathrm{~m} / \mathrm{s})\end{array}$ & $\begin{array}{l}\mathrm{I} / \mathrm{N} 8 \\
(\mathrm{~m} / \mathrm{s})\end{array}$ & $\begin{array}{l}\text { Anchorage } \\
(\mathrm{m} / \mathrm{s})\end{array}$ & $\begin{array}{l}\mathrm{I} / \mathrm{N} 1-3 \\
(\mathrm{~m} / \mathrm{s})\end{array}$ & $\begin{array}{l}\mathrm{I} / \mathrm{N} 5 \\
(\mathrm{~m} / \mathrm{s})\end{array}$ & $\begin{array}{l}\mathrm{I} / \mathrm{N} 8 \\
(\mathrm{~m} / \mathrm{s})\end{array}$ \\
\hline 4 & 0 & 8.52 & 11.85 & 8.68 & 8.47 & 8.95 & 9.44 & 6.74 & 4.63 \\
\hline 4 & 100 & 8.40 & 11.84 & 8.47 & 8.80 & 9.35 & 8.43 & 6.10 & 4.65 \\
\hline 4 & 200 & 8.62 & 11.06 & 8.21 & 8.12 & 9.13 & 8.95 & 6.22 & 4.33 \\
\hline 6 & 0 & 8.08 & 11.10 & 7.48 & 6.96 & 8.93 & 8.37 & 5.77 & 4.25 \\
\hline 6 & 100 & 7.63 & 10.29 & 7.62 & 7.08 & 8.76 & 7.93 & 5.81 & 3.94 \\
\hline 6 & 200 & 7.64 & 10.66 & 7.73 & 6.83 & 8.33 & 7.55 & 5.37 & 3.71 \\
\hline 12 & 0 & 6.54 & 7.80 & 5.33 & 4.72 & 8.04 & 7.07 & 4.91 & 3.43 \\
\hline 12 & 100 & 6.50 & 8.05 & 5.24 & 4.64 & 8.36 & 7.07 & 4.56 & 3.24 \\
\hline 12 & 200 & 6.50 & 7.76 & 5.23 & 5.19 & 8.21 & 7.00 & 4.57 & 3.14 \\
\hline 4 & mean & 8.51 & 11.58 & 8.45 & 8.46 & 9.14 & 8.94 & 6.35 & 4.54 \\
\hline 6 & mean & 7.79 & 10.68 & 7.61 & 6.96 & 8.68 & 7.95 & 5.65 & 3.97 \\
\hline 12 & mean & 6.51 & 7.87 & 5.27 & 4.85 & 8.21 & 7.05 & 4.68 & 3.27 \\
\hline mean & 0 & 7.71 & 10.25 & 7.16 & 6.72 & 8.64 & 8.29 & 5.81 & 4.10 \\
\hline mean & 100 & 7.51 & 10.06 & 7.11 & 6.84 & 8.82 & 7.81 & 5.49 & 3.94 \\
\hline mean & 200 & 7.59 & 9.83 & 7.06 & 6.71 & 8.56 & 7.83 & 5.39 & 3.73 \\
\hline Plants $/ \mathrm{m}^{2}$ & SED & $0.095 * * *$ & $0.482 * * *$ & $0.157 * * *$ & $0.229 * * *$ & $0.180 * * *$ & $0.297 * * *$ & $0.186^{* * *}$ & $0.172 * * *$ \\
\hline Nitrogen & SED & 0.095 & 0.482 & 0.157 & 0.229 & 0.180 & 0.297 & 0.186 & 0.172 \\
\hline Interaction & SED & 0.165 & 0.835 & 0.271 & 0.397 & 0.312 & 0.514 & 0.323 & 0.298 \\
\hline
\end{tabular}


Table 8. CH18: Stem and Anchorage failure wind speeds

\begin{tabular}{|c|c|l|l|l|l|}
\hline Plants/m & $\begin{array}{l}\mathrm{N} \text { rate } \\
\mathrm{kg} / \mathrm{ha}\end{array}$ & $\begin{array}{l}\text { Anchorage } \\
(\mathrm{m} / \mathrm{s})\end{array}$ & $\begin{array}{l}\mathrm{I} / \mathrm{N} 1-3 \\
(\mathrm{~m} / \mathrm{s})\end{array}$ & $\begin{array}{l}\mathrm{I} / \mathrm{N} 5 \\
(\mathrm{~m} / \mathrm{s})\end{array}$ & $\begin{array}{l}\mathrm{I} / \mathrm{N} 8 \\
(\mathrm{~m} / \mathrm{s})\end{array}$ \\
\hline 5.5 & 60 & 7.58 & 11.62 & 8.87 & 6.30 \\
\hline 5.5 & 180 & 7.28 & 11.55 & 9.49 & 6.58 \\
\hline 5.5 & 300 & 7.25 & 12.13 & 9.79 & 7.52 \\
\hline 7 & 60 & 7.45 & 12.06 & 8.33 & 6.18 \\
\hline 7 & 180 & 7.06 & 11.66 & 8.63 & 6.96 \\
\hline 7 & 300 & 6.87 & 11.69 & 7.89 & 6.80 \\
\hline 8.5 & 60 & 7.44 & 11.79 & 7.31 & 5.68 \\
\hline 8.5 & 180 & 6.87 & 11.67 & 7.46 & 6.08 \\
\hline 8.5 & 300 & 6.78 & 10.97 & 7.12 & 5.99 \\
\hline 10 & 60 & 7.02 & 10.70 & 7.20 & 5.76 \\
\hline 10 & 180 & 6.75 & 10.19 & 6.59 & 5.67 \\
\hline 10 & 300 & 6.65 & 9.75 & 6.29 & 5.50 \\
\hline & & & & & \\
\hline 5.5 & mean & 7.37 & 11.8 & 9.38 & 6.80 \\
\hline 7 & mean & 7.13 & 11.8 & 8.29 & 6.64 \\
\hline 8.5 & mean & 7.03 & 11.5 & 7.29 & 5.92 \\
\hline 10 & mean & 6.81 & 10.2 & 6.69 & 5.64 \\
\hline mean & 60 & 7.37 & 11.5 & 7.93 & 5.98 \\
\hline mean & 180 & 6.99 & 11.3 & 8.04 & 6.32 \\
\hline mean & 300 & 6.89 & 11.1 & 7.77 & 6.45 \\
\hline & & & & & \\
\hline Plants/m & SED & $0.089^{* *}$ & $0.382^{*}$ & $0.487 * *$ & 0.362 \\
\hline Nitrogen & SED & $0.048^{* * *}$ & 0.374 & 0.245 & 0.207 \\
\hline Interaction & SED & 0.119 & 0.721 & 0.631 & 0.495 \\
\hline & & & & & \\
\hline
\end{tabular}

\subsection{Predicting lodging associated lodging characters}

295 Increasing the plant population increased the leaf area index (LAI) at the four, six and eight leaf stages in both the UK17 and UK18 field experiments and at the six and eight leaf stages in the $\mathrm{CH} 18$ experiment $(\mathrm{P}<0.001$; Tables 9 and 10). Increasing fertiliser rate from 60 to 180 $\mathrm{kg} \mathrm{N} /$ ha caused a modest, but significant $(\mathrm{P}<0.05)$, increase in LAI of 0.34 units at Leaf 8 in the $\mathrm{CH} 18$ experiment, but there was no evidence that increasing the $\mathrm{N}$ fertiliser rate affected

300 LAI between the four and eight leaf stages in the UK experiments.

In all experiments LAI measured at the four, six or eight leaf stage was positively correlated with biomass yield at harvest, the combined lengths of the bottom three internodes and the plant's natural frequency, negatively correlated with stem diameter, stem failure moment, 
root plate dimensions measured after flowering and the calculated stem and root failure wind speeds ( $\mathrm{P}<0.05$; Table 11). The correlations were generally stronger in the UK17

307 flowering and the length of the lower internodes.

309 Table 9. Leaf area index measured at 4, 6 and 8 leaf stages in the UK17 and UK18 310 experiments.

\begin{tabular}{|c|c|l|l|l|l|l|l|}
\hline & & \multicolumn{3}{|c|}{ UK17 } & \multicolumn{3}{c|}{ UK18 } \\
\hline Plants/m2 & $\begin{array}{l}\text { N } \\
\text { applied } \\
\text { kg/ha }\end{array}$ & Leaf 4 & Leaf 6 & Leaf 8 & Leaf 4 & Leaf 6 & Leaf 8 \\
\hline 4 & 0 & 0.100 & 1.03 & 1.38 & 0.0243 & 0.553 & 2.29 \\
\hline 4 & 100 & 0.100 & 0.87 & 1.23 & 0.0242 & 0.637 & 2.67 \\
\hline 4 & 200 & 0.103 & 0.85 & 1.28 & 0.0243 & 0.634 & 1.77 \\
\hline 6 & 0 & 0.158 & 1.51 & 1.81 & 0.0554 & 1.121 & 2.63 \\
\hline 6 & 100 & 0.149 & 1.36 & 1.89 & 0.0554 & 0.829 & 2.82 \\
\hline 6 & 200 & 0.170 & 1.44 & 1.72 & 0.0549 & 1.043 & 2.97 \\
\hline 12 & 0 & 0.319 & 2.71 & 3.46 & 0.2206 & 1.686 & 3.74 \\
\hline 12 & 100 & 0.302 & 2.71 & 3.54 & 0.2387 & 1.519 & 4.53 \\
\hline 12 & 200 & 0.329 & 2.68 & 3.03 & 0.1982 & 1.551 & 4.30 \\
\hline & & & & & & & \\
\hline 4 & mean & 0.101 & 0.92 & 1.30 & 0.0243 & 0.608 & 2.24 \\
\hline 6 & mean & 0.159 & 1.44 & 1.81 & 0.0552 & 0.998 & 2.81 \\
\hline 12 & mean & 0.317 & 2.70 & 3.34 & 0.2192 & 1.585 & 4.19 \\
\hline mean & 0 & 0.192 & 1.75 & 2.22 & 0.1001 & 1.120 & 2.89 \\
\hline mean & 100 & 0.184 & 1.65 & 2.22 & 0.1061 & 0.995 & 3.34 \\
\hline mean & 200 & 0.201 & 1.66 & 2.01 & 0.0925 & 1.076 & 3.01 \\
\hline & & & & & & & \\
\hline Plants/m ${ }^{2}$ & SED & $0.0071 *$ & $0.091 * * *$ & $0.217 * * *$ & $0.00774 * * *$ & $0.0592^{* * *}$ & $0.237^{* * *}$ \\
\hline Nitrogen & SED & 0.0071 & 0.091 & 0.217 & 0.00774 & 0.0592 & 0.237 \\
\hline Interaction & SED & 0.0123 & 0.157 & 0.375 & 0.01341 & 0.1026 & 0.410 \\
\hline
\end{tabular}

312 Table 10. Leaf area index measured at 6 and 8 leaf stages in the $\mathrm{CH} 18$ experiment.

\begin{tabular}{|c|c|l|l|}
\hline Plants/m2 & $\begin{array}{l}\mathrm{N} \\
\text { applied } \\
\mathrm{kg} / \mathrm{ha}\end{array}$ & Leaf 6 & Leaf 8 \\
\hline 5.5 & 60 & 0.198 & 0.939 \\
\hline 5.5 & 180 & 0.282 & 1.065 \\
\hline 5.5 & 300 & 0.313 & 1.135 \\
\hline 7 & 60 & 0.277 & 1.191 \\
\hline 7 & 180 & 0.397 & 1.470 \\
\hline 7 & 300 & 0.426 & 1.568 \\
\hline
\end{tabular}




\begin{tabular}{|c|c|c|c|}
\hline 8.5 & 60 & 0.363 & 1.379 \\
\hline 8.5 & 180 & 0.487 & 1.914 \\
\hline 8.5 & 300 & 0.574 & 1.905 \\
\hline 10 & 60 & 0.410 & 1.550 \\
\hline 10 & 180 & 0.524 & 1.962 \\
\hline 10 & 300 & 0.645 & 2.179 \\
\hline 5.5 & mean & 0.264 & 1.05 \\
\hline 7 & mean & 0.367 & 1.41 \\
\hline 8.5 & mean & 0.475 & 1.73 \\
\hline 10 & mean & 0.526 & 1.90 \\
\hline mean & 60 & 0.312 & 1.26 \\
\hline mean & 180 & 0.423 & 1.60 \\
\hline mean & 300 & 0.490 & 1.70 \\
\hline Plants/m $\mathrm{m}^{2}$ & SED & $0.0359 * * *$ & $0.0814 * * *$ \\
\hline Nitrogen & SED & $0.0101 * * *$ & $0.0721 * * *$ \\
\hline Interaction & SED & $0.0395 * *$ & 0.1432 \\
\hline
\end{tabular}

315 Table 11. Correlation coefficients calculated for leaf area index measured at leaf(L) 4, 6 \& 8

316 with plant characteristics associated with lodging and biomass yield. White cells represent

317 strong positive correlations and dark grey cells represent strong negative correlations.

Biomass yield

Crop height

Height at centre of gravity

Natural Frequency

Length internodes 1-3

Length Internode 5

Length internode 8

Diameter internode 2

Diameter Internode 5

Diameter internode 8

Wall width internode 2

Wall width Internode 5

Wall width internode 8

Failure moment internodes 1-3

Failure moment Internode 5

Failure moment internode 8

Root plate spread

Root plate depth

Failure wind speed internode 2
UK17

L4 L6

$0.90 \quad 0.92$

$0.91 \quad 0.95$

$0.97 \quad 0.96$

$-0.87$

0.6

$0.88 \quad 0.91$

$0.19 \quad 0.30$

$-0.96-0.96$

$-0.74-0.74$

$-0.93 \quad-0.90$

$-0.77-0.75$

$\begin{array}{ll}-0.20 & -0.19\end{array}$

$-0.12 \quad-0.05$

$\begin{array}{ll}-0.77 & -0.69\end{array}$

$\begin{array}{ll}-0.93 & -0.88\end{array}$

$-0.85-0.84$

$-0.84-0.86$

$-0.68-0.64$

$-0.89-0.83$
UK18

$\mathrm{CH} 18$

$\begin{array}{llllll}\text { L8 } & \text { L4 } & \text { L6 } & \text { L8 } & \text { L6 } & \text { L8 }\end{array}$

\begin{tabular}{|l|l|l|l|l|l}
0.84 & 0.54 & 0.44 & 0.48 & 0.70 & 0.66
\end{tabular}

$\begin{array}{lllllll}0.87 & 0.03 & 0.01 & -0.04 & 0.57 & 0.50\end{array}$

0.91

0.11

0.11

0.01

0.70

0.64

$-0.46$

0.40

$-0.38$

$-0.38$

0.24

$-0.90 \quad-0.87$

0.70

$\begin{array}{rr}0.22 & 0.25\end{array}$

0.11

0.80

0.85

0.74

$-0.15$

$-0.05$

$-0.13$

0.40

0.35

$-0.73-0.76$

$-0.64$

0.75

0.67

$\begin{array}{ll}-0.15 & -0.25\end{array}$

$-0.71$

$-0.63$

$-0.62$

$-0.63$

$-0.07$

$-0.19$

$-0.88$

$-0.69$

$-0.67$

$-0.59$

$-0.19$

$-0.23$

$-0.72$

$\begin{array}{ll}-0.62 & -0.70\end{array}$

$-0.57$

$-0.02$

$-0.08$

$-0.22$

$-0.76$

$-0.71$

$-0.55$

$-0.13$

$-0.24$

0.07

$-0.25$

$-0.31$

$-0.09$

0.55

0.45

$-0.81$

$-0.66$

$-0.63$

$-0.65$

$-0.24$

$-0.27$

$-0.57$

$-0.78$

$-0.70$

$-0.70$

$-0.66$

$-0.49$

$-0.27$

$-0.78$

$-0.76$

$-0.70$

$-0.72$

$-0.18$

$-0.41$

$-0.72$

$\begin{array}{ll}-0.71 & -0.64\end{array}$

$-0.66$

$-0.39$

$-0.47$

$-0.74$

$-0.73$

$-0.68$ $-0.74$ 
Failure wind speed Internode 5

Failure wind speed internode 8 318

319

320

321

322

323

324

325

326

327

328

329

330

331

332

333

334

335

336

337

\section{Discussion}

The model of the process of maize lodging has been evaluated in UK and China environments. The lodging model described in this paper calculates failure wind speeds of lower internodes 2 and 5 of between 5 and $12 \mathrm{~m} / \mathrm{s}$, failure wind speeds of internode 8 of 3 to $7 \mathrm{~m} / \mathrm{s}$ and root failure wind speeds for soil at field capacity water content of 6 to $9 \mathrm{~m} / \mathrm{s}$. These calculated failure wind speeds represent average wind velocities at crop height. The gust values at crop height that are likely to cause lodging will be $2-2.5$ times greater than these values. The failure wind speed values can also be extrapolated to the normal meteorological measurement height of $10 \mathrm{~m}$ above ground level through the use of the logarithmic velocity profile using the method described by Joseph et al. (2020) From this a failure wind speed of 4 $\mathrm{m} / \mathrm{s}$ extrapolates to a velocity at $10 \mathrm{~m}$ height of approximately $12 \mathrm{~m} / \mathrm{s}$. For context, in the UK, the 99th percentile average hourly wind speed at $10 \mathrm{~m}$ height is between 11 and $13 \mathrm{~m} / \mathrm{s}$ (Cook, 1985). Therefore, the failure wind speeds calculated for the maize crops grown in the field trials would only be expected to be exceeded a small proportion of the time. None of the field trials described in the study experienced natural lodging which indicates that the calculated failure wind speeds are realistic for the test crops in question.

The root failure wind speeds when the soil is at field capacity are less than, or greater than, the failure wind speed of the lower internode 2, depending on the experiment. This implies that stem or root lodging may be most prevalent depending on the characteristics of the plant and the conditions it experiences. This is consistent with detailed observations of maize lodging which have shown both stem and root lodging to be possible (Hu et al., 2013; Fincher 
et al. 1985; Kamara et al. 2003). The calculated stem failure wind speeds are greatest for the lower internode 2 and decline by more than half for the middle internode 8 . This is because the strength of internode 8 was measured to be less than one quarter of the strength of internode 2 , but the force that internode 8 must support was calculated to be more than one quarter of the force that internode 2 must support. At this point it should be recognised that towards the top of the plant, the stem will be more flexible, and will be the part of the crop that behaves least like the bending cantilever assumed in the lodging model. We would therefore expect the model to predict lower stem failure wind speeds with greater reality than for upper parts of the stem and possibly also the mid stem. Further research is required to test this part of the model. For the lower internodes, for which the model assumptions are most applicable, the model predicts that failure at internode 5 is more likely than failure of internode 2 in all the experiments. Observations of natural lodging have shown that stem failure can be at any point between the soil and the cob (Arnold and Josephson, 1975) and can therefore occur on the bottom or middle internodes.

The maize lodging model calculated that increasing plant population substantially reduced the stem and root failure wind speeds, thus increasing the likelihood of lodging. Increasing plant density from what may be regarded commercially as low (6 plants $\left./ \mathrm{m}^{2}\right)$ to high $(10-12$ plants $/ \mathrm{m}^{2}$ ) plant densities was estimated to reduce the stem and root failure wind speeds by 1results in a two order of magnitude change in risk. These predictions for increased plant density to cause a substantial increase in lodging risk are consistent with observations of plant population effects on natural lodging (Guo et al., 2019; Sher et al., 2017; Jun et al., 2017).

Previous studies have shown that increasing $\mathrm{N}$ fertiliser either has little effect on lodging risk or increases resistance to stem lodging by increasing stem diameter (Peng et al. 2014; Shi et 
al., 2016). These findings are also consistent with the prediction of the effect of $\mathrm{N}$ fertiliser on lodging made by the maize lodging model described in this present study.

Investigation of maize anchorage by this study demonstrated that the spread of the root plate is a key determinant of anchorage strength similar to that used in a model of wheat lodging by Berry et al. (2003). This is consistent with Ennos et al. (1993) who concluded that the mechanism of anchorage failure in maize was similar to wheat. The angle of root spread has also been shown to be a key criterion for determining varietal differences in maize anchorage strength (Liu et al., 2012). Other root characteristics have been shown to be superior predictors of anchorage strength in maize including the biomass of the roots in the upper region of soil by this present study and the number and thickness of crown roots (Liu et al., 2012). However, it should be recognised that these rooting characteristics require much more time to measure than the spread of the root plate, which limits their utility as a screening method for anchorage strength.

A sensitivity analysis of the key traits that determine the wind induced shoot leverage (natural frequency), anchorage strength (root plate spread) and stem strength itself showed that stem failure wind speed was influenced more by variation in stem strength than by variation in shoot natural frequency and root failure wind speed was influenced more by variation in the spread of the root plate than by variation in shoot natural frequency (Figure 4). Therefore plant breeders should focus on increasing stem strength and anchorage strength to achieve the greatest increase in lodging resistance. Measuring stem strength and shoot natural frequency are quite laborious, so it will be useful to identify surrogate measures that provide a reasonable approximation of these traits, particularly for stem strength. Stem diameter and stem strength were generally closely related ( $\mathrm{R}=0.55$ to 0.86$)$. Crop height was also generally well correlated with natural frequency $(\mathrm{R}=-0.50$ to -0.87$)$. Both stem diameter and crop height are simple traits to measure which increases their utility as a screening tool. Root plate 
391

392

393

394

395

396

397

398

399

400

401

402

spread, the key component of root failure wind speed, is less easy to measure because the plant must be excavated, however it may be possible to develop a procedure to assess this trait without washing the soil from the roots which would reduce the measurement time substantially. Further research is required to assess whether a simplified lodging model can be developed which uses the key 'easy-to-measure' traits (crop height, stem diameter and root plate spread) and provides a sufficiently reliable estimate of lodging risk. The correlation analysis also reveals that the maize biomass yield was negatively correlated with lodging resistance (stem and root failure wind speed) $(\mathrm{R}=-0.24$ to -0.88$)$. This illustrates the important trade-off that maize growers and advisors must consider when choosing the optimum combination of agronomic treatments. at 
root failure wind speed: natural frequency (open circles, solid line), stem strength (closed circles, solid line).

This study has demonstrated that the LAI measured at leaf 4,6 or 8 is a good indicator of a crop's risk of lodging later in the growing season. This relationship is mainly caused by variation in plant population which has a strong effect on both early LAI and the lodging associated plant characters that have a strong influence on the calculation of stem and root failure wind speed such as stem strength and root plate spread. Regression analysis of the LAI measured at leaf 6 and the failure wind speed of internode 5 showed that the best model fit was parallel lines with different $Y$ intercepts $\left(P<0.001, R^{2}=0.86\right.$; Figure 5). An increase in the LAI measured at leaf 6 of one unit corresponded to a reduction in the failure wind speed of internode 5 of approximately $2 \mathrm{~m} / \mathrm{s}$ (Figure 5). In 2018, the crop experienced water stress at Leaf 6 which may explain why the relationship in this experiment was different to the other experiments. This analysis indicates that early measurements of canopy size could be used to predict the risk of lodging in time for the application of remedial treatments such as plant growth regulators that have been shown to reduce lodging risk by reducing the rate of stem extension and final crop height (Shekoofa and Emam, 2008; Spitzer et al., 2015). Plant growth regulators are effective when applied at the 8-9 leaf stage and later at the 4-6 detectable node stage (Spitzer et al., 2015), therefore predicting lodging risk at the 4 to 8 leaf stage will be early enough to make decisions about whether to apply this treatment. The similar sensitivity between experiments of the failure wind speed to changes in early LAI suggests that early LAI could be used to quantify intra-field variation in lodging risk. This could form the basis for spatially varying the rates of remedial treatments according to variation in lodging risk. However, the different $\mathrm{Y}$ intercepts between the experiments means further research is required to develop a quantitative prediction between fields. Additional information such as variety maybe required to achieve this. Leaf area index is laborious to 
measure, but the use of spectral indices recorded by remote sensing devices should provide an efficient alternative for measuring LAI repeatedly over large spatial extents (Xia et al., 2016). The concept of spatially of varying plant growth regulator applications within fields based on remotely sensed information about canopy size has been demonstrated in wheat by (Griffin and Hollis, 2017).

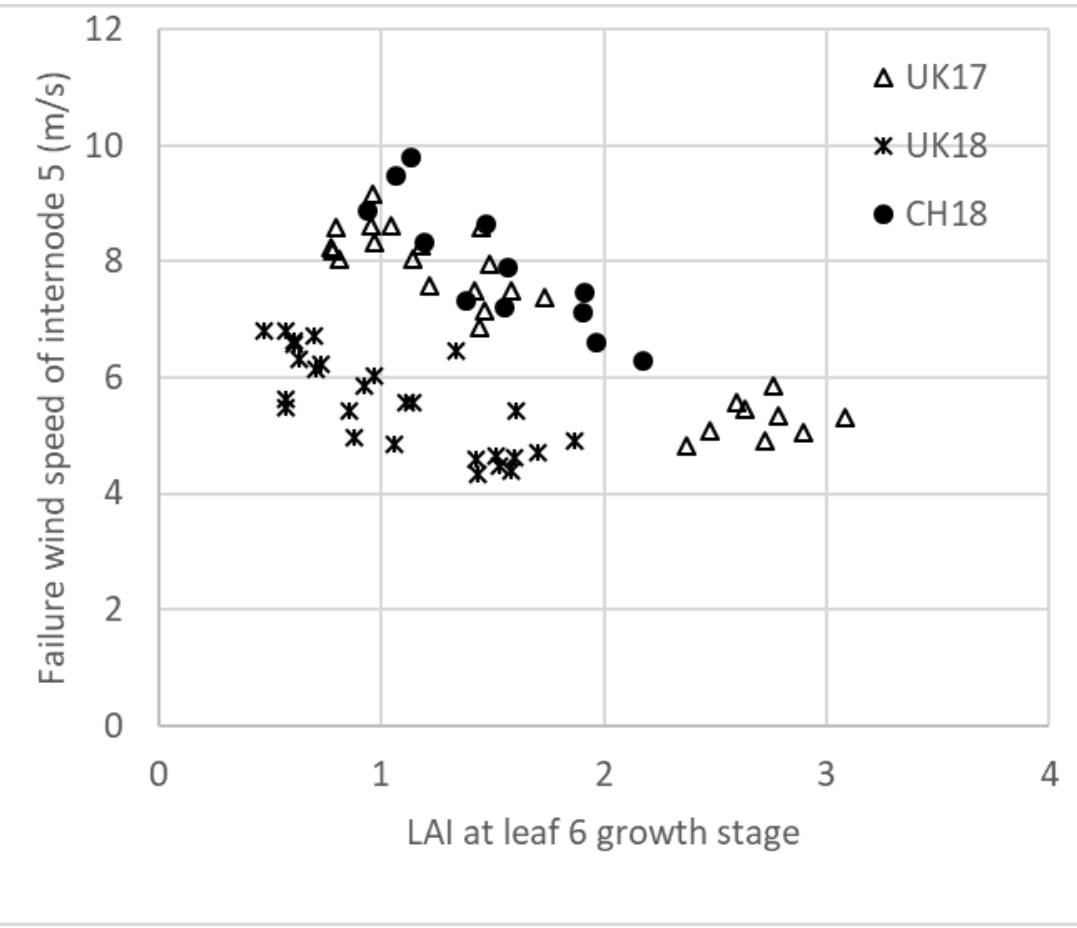

Figure 5. Relationship between the failure wind speed of internode 5 at tasselling and the LAI measured at when leaf 6 had fully expanded.

After further validation the maize lodging model described in this paper could form the basis of a decision support system that helps farmers and crop advisors to strategically plan crop management systems that maximises productivity and minimises lodging risk. Predictions of lodging risk based on the status of the developing crop, using measures such as LAI, will help farmers to tactically fine-tune crop husbandry and take account of early season growing conditions. A framework for a crop lodging decision support system known as CROPFALL has been described by Berry et al. (2019). This decision support system is designed to 
integrate a lodging model with remote sensing information to inform crop husbandry decisions on a field-by-field basis and on a metre-by-metre basis, thereby enabling precision application of crop inputs.

\section{Conclusions}

The model of maize lodging risk described in this paper has been shown to calculate plausible failure wind speeds and the model's calculations of the effects of plant population and $\mathrm{N}$ fertiliser on lodging risk were consistent with published observations. Further work is required to test the model's output against observations of natural lodging, but the results give confidence that the lodging model can be used to better understand the effect of crop husbandry decisions on lodging risk. This will help farmers and crop advisors to develop crop husbandry strategies for minimising lodging risk. Leaf area index measured at leaf 4, 6 or 8 stages was shown to be a good indicator of the future values of the plant characters associated with lodging that are used as the lodging model inputs. This opens up the potential to develop the lodging model to predict lodging risk early enough in the growing season to allow farmers to make tactical agronomic decisions to minimise lodging risk. A sensitivity analysis showed that variation in stem strength and the spread of the root plate are the most important characteristics that influence the risk of stem and root lodging, therefore plant breeding and crop husbandry should focus on maximising these traits.

\section{Acknowledgements}

This study was made possible by funding grants from the British Biology and Biosciences Research Council Global Challenges Research Fund (GCRF BB/P023282) and the Sustainable Agriculture Research and Innovation Club (SARIC BB/P004555) fund. 


\section{References}

473 Arnold, J. M. and Josephson, L. M., 1975. Inheritance of stalk quality characteristics in maize. Crop Sci. 15, 338-340.

475 http://dx.doi.org/10.2135/cropsci1975.0011183X001500030017x

Baker, C.J., Berry, P.M., Spink, J.H., Sylvester-Bradley, R., Griffin, J.M., Scott, R.K. and

477 Clare, R.W., 1998. A method for the assessment of the risk of wheat lodging. Journal of 478 Theoretical Biology 194, 587-603.

Baker, C.J., Sterling, M. and Berry, P.M. 2014. A generalised model of lodging. Journal of 480 Theoretical Biology 363, 1-12. doi: 10.1016/j.jtbi.2014.07.032.

Berry, P.M., Griffin, J.M., Sylvester-Bradley, R., Scott, R.K., Spink, J.H., Baker, C.J., and Clare, R.W., 2000. Controlling plant form through husbandry to minimise lodging in wheat. Field Crops Research 67, 59-81.

Berry, P.M., Sterling, M., Baker, C.J., Spink, J.H., Sparkes, D.L., 2003. A calibrated model of wheat lodging compared with field measurements. Agricultural and Forest Meteorology. 119, 167-180.

Berry, P.M., Sterling, M., Spink, J.H. Baker, C.J., Sylvester-Bradley, R., Mooney, S., Tams, A., Ennos, A.R. 2004. Understanding and reducing lodging in cereals. Advances in Agronomy 84, 215-269.

Berry, P.M. and Spink, J.H., 2009. Understanding the effect of a triazole with anti-gibberellin

491 activity on the growth and yield of oilseed rape (Brassica napus). Journal of Agricultural

492 Science, Cambridge 147, 273-285. 
Berry, P., Blackburn, A., Sterling, M., Miao, Y., Hatley, D., Gullick, D., Joseph, G., Whyatt, D., Soper, D., Murray, J., Baker, C., 2019. A multi-disciplinary approach for the precision management of lodging risk. 12th European Conference of Precision Agriculture, Montpellier, p683-687 Wageningen Academic Publishers.

497

Colbert, T.R., Darrah, L.L., Zuber, M.S., 1984. Effect of recurrent selection for stalk crushing strength on agronomic characteristics and soluble stalk solids in maize. Crop Science, 24,

499 $473-478$.

500 Cook, N.J., 1985. The designer's guide to wind loading on building structures. Part 1, BRE, 501 Butteworths.

Ennos, A.R., Crook, M.J., Grimshaw, C., 1993. The anchorage mechanics of maize (Zea mays). Journal of Experimental Botany 44, 147-153.

504

Fincher, R.R., Darrah, L.L., Zuber, M.S., 1985. Root development in maize as measured by vertical root-pulling resistance. Maydica, 30, 383-394.

Flint-Garcia, S.A., Jampatong, C., Darrah, L.L., McMullen, M.D., 2003. Quantitative trait

507 locus analysis of stalk strength in four maize populations. Crop Sci. 43:13-22. http//dx.doi.org/10.2135/cropsci2003.0013

509 Fouéré, A., Pellerin, S., Duparque, A., 1995. A portable electronic device for evaluating root 510 lodging resistance in maize. Agronomy Journal, 87, 1020-1024.

511 Griffin, S. and Hollis, J. (2017). Plant growth regulators on winter wheat - yield benefits of variable rate application. Advances in Animal Biosciences: Precision Agriculture (ECPA) 2017, (2017), 8:2, pp 233-237 doi:10.1017/S2040470017000267. 

stalk lodging resistance using equivalent forces combined with SVD algorithm. Appl. Sci., 9, 640; http//dx.doi.org:10.3390/app9040640 A.E., Chen, S., 2013. QTL mapping of stalk bending strength in a recombinant inbred line maize population. Theor. Appl. Genet. 126,2257-2266

Huang, L., Qiao, J.F., Liu, J.B., Xia, L.K., Zhu, W.H., Li, C., Zhou, Q.W., 2015. Research on the relationship between maize lodging resistance and grain mechanically harvesting qualities in different planting density. Acta Agriculturae Boreali-Sinica, 30, 198-201. (Mandarin) M., Blackburn, A., Whyatt, D., Gullick, D., Murray, J., Berry, P., Hatley, D., Finnan, J., 2020. Determination of crop dynamic and aerodynamic parameters for lodging prediction. Journal of Wind Engineering \& Industrial Dynamics 202, https://dx.doi.org/10.1016/j.jweia.2020.104169. Jun, X., Rui-zhi, X., Wang-feng, Z., Ke-ru, W., Peng, H., Bo, M., Ling, G., Shaokun. L., 2017. Research progress on reduced lodging of high-yield and -density maize. Journal of Integrative Agriculture 2017, 16(12): 2717-2725. pulling resistance with root lodging and grain yield in selected S1 maize lines derived from a tropical low nitrogen population. Journal of Agronomy \& Crop Science, 189, 129-135. recombinant inbred line populations. BMC Plant Biol. 14, 152. 
Li, S.Y., Ma, W., Peng, J.Y., Chen, Z. M., 2015a. Study on yield loss of summer maize due to lodging at the big flare stage and grain filling stage. Scientia Agricultura Sinica, 19, 39523964. (Chinese text)

Li S Y, Wang Y X, Hu C D, Yan Y. 2015b. Effect of strong wind lodging at pre- and posttasseling stages on growth and yield of summer maize. Chinese Journal of Applied Ecology, 26, 2405-2413. (Chinese text)

Liu, S., Song, F., Liu, F., Zhu, X., Xu, H., 2012. Effect of Planting Density on Root Lodging Resistance and Its relationship to Nodal Root Growth Characteristics in Maize (Zea mays L.). Journal of Agricultural Science 4, 182-189.

Mohammadi, R., Finnan, J., Sterling, M. and Baker C. J., 2020. A calibrated oat lodging model compared with agronomic measurements. Field Crops Research. 225107784. http://doi.org.10.1016/j.fcr.2020.107784.

Peng, D.L., Chen, X.G., Yin, Y.P., Lu, K.L., Yang, W.B., Tang, Y.H., Wang, Z.L., 2014. Lodging resistance of winter wheat (Triticum aestivum L.): Lignin accumulation and its related enzymes activities due to the application of paclobutrazol or gibberellin acid. Field Crops Research, 157: 1-7.

Shengqun, Liu., Fengbin, Song., Fulai, Liu., Xiancan, Zhu., Haibo, Xu., 2012. Effect of Planting Density on Root Lodging Resistance and Its Relationship to Nodal Root Growth Characteristics in Maize (Zea mays L.) Journal of Agricultural Science; Vol. 4, No. 12; 2012. Sher, A., Khan, A., Jin Cai, L., Ahmad, M., Asharf, U. and Ali Jamoro, S., 2017. Response of Maize Grown Under High Plant Density; Performance, Issues and Management - A Critical Review. Advances in Crop Science and Technology, 5, 1-8. http://doi.org/10.4172/23298863.1000275 . 
Shi, D., Li, Y., Zhang, J., Liu, P., Zhao, B., Dong, S., 2016. Effects of plant density and nitrogen rate on lodging-related stalk traits of summer maize. Plant Soil Environment 62, 299-306.

Shekoofa, A., Emam, Y., 2008. Plant growth regulator (ethephon) alters maize (Zea Mays L.) growth, water use and grain yield under water stress. Journal of Agronomy 7, 41-48.

Spitzer, T., Misa, P., Bilovsky, J., Kazda, J., 2015. Management of Maize Stand Height using Growth Regulators. Plant Protection Science 51, 223-230.

Sposaro, M.M., Chimenti, C.A., Hall, A.J., 2008. Root lodging in sunflower. Variations in anchorage strength across genotypes, soil types, crop population densities and crop developmental stages. Field Crops Res. 106, 179-186.

Sporoso, M.M., Berry, P.M., Sterling, M., Hall, A.J., Chimenti, C.A., 2010. Modelling root and stem lodging in sunflowers. Field Crops Research 119, 125-134.

Wen, W., Gu, S., Xiao, B., Wang, C., Wang, J., Ma, L., Wang, Y., Lu, X., Yu, Z., Zhang, Y., Du, J., Guo, X., 2019. In situ evaluation of stalk lodging resistance for different maize (Zea mays L.) cultivars using a mobile wind machine. Plant Methods, 15, 1-16. http://dx.doi.org/10.1186/s13007-019-0481-1.

Xia, T., Miao, Y., Wu, D., Shao, H., Khosla, R., M, G., 2016. Active optical sensing of spring maize for in-season diagnosis of nitrogen status based on nitrogen nutrition index. Remote Sensing, 8(7), 605 .

Xue, J., Xie, R., Zhang, W., Wang, K., Hou, P, Ming, B., Gou, L., Li, S., 2017. Research progress on reduced lodging of high-yield and -density maize. Journal of Integrative Agriculture. 16(12): 2717-2725. 